\title{
DETERMINAÇÃO DA VELOCIDADE ECONÔMICA EM ADUTORAS SOB DIFERENTES CONDIÇÕES DE CUSTO E FUNCIONAMENTO
}

André Luiz Dias Caldas ${ }^{1}$, Jacinto de Assunção Carvalho², Fátima Conceição Rezende ${ }^{3}$

\section{RESUMO}

Visando maior eficiência em projetos de adutoras para irrigação, vários estudos são realizados, principalmente, sobre as características hidráulicas desses sistemas. No entanto, mesmo que hidraulicamente correto, o projeto pode não atender aspectos econômicos. Sobre este contexto, o presente trabalho teve por objetivo fazer um estudo hidráulico e econômico sobre a velocidade de escoamento da água em adutoras de aço galvanizado e PVC. Foi considerado uma adutora padrão, composta pela tubulação de sucção, conjunto motobomba, sistema de ligação e proteção e tubulação de recalque, operando com vazões de 50,100, 150, 200, 250 e $300 \mathrm{~m}^{3} \mathrm{~h}^{-1}$ e pressões nominais de 80,125 e $180 \mathrm{mca}$, em função do tempo de funcionamento, desnível geométrico total, custo de energia de bombeamento e de aquisição da tubulação. Verificou-se que para tubulações de aço galvanizado a velocidade econômica variou entre 0,88 e $2,65 \mathrm{~m} \mathrm{~s}^{-1}$ e para tubulações de PVC, entre 0,68 e 2,54 $\mathrm{m} \mathrm{s}^{-1}$. Pode-se concluir que o valor da velocidade econômica é decrescente com o aumento no tempo de funcionamento da adutora e com o aumento no desnível geométrico total. A velocidade econômica foi indiretamente influenciada pelo aumento na tarifa energética e diretamente influenciada pelo aumento no custo da tubulação.

Palavras-chave: Conduto forçado, Dimensionamento econômico, Energia elétrica.

\begin{abstract}
DETERMINATION OF ECONOMIC SPEED AQUEDUCTS UNDER DIFFERENT CONDITIONS OF COST AND OPERATION

Aiming higher efficiency in irrigation pipelines projects several studies are done mainly about hydraulic characteristics of these systems. However, even hydraulically that's right, the project can't answer economic aspects. This context the working gift aimed make hum hydraulic and economic study on the flow rate of the water mains in galvanized steel and PVC. It was considered a standard pipeline, composed by suction pipe, pump set, connection system and protection and discharge piping, operating with flow rates of 50,100, 150, 200, 250 and $300 \mathrm{~m}^{3} \mathrm{~h}^{-1}$ and nominal pressures of 80, 125 and $180 \mathrm{mca}$, operating time function, the total geometric drop, energy cost of pumping and piping of the acquisition. There was that steel pipes galvanized the economic speed varied among 0.88 and $2.65 \mathrm{~m}$ $\mathrm{s}^{-1}$ and PVC pipes, between 0.68 and $2.54 \mathrm{~m} \mathrm{~s}^{-1}$. It can be concluded that value of economic speed and decreases with the increase in the rate of operation of the pipeline and the increase in total drop geometric. The economic speed was indirectly influenced hair increase in energy tariff and directly influenced hair increased pipe cost.
\end{abstract}

Keywords: Pipe under pressure, Economic sizing, Electric power.

\section{Recebido para publicação em 10/03/2015. Aprovado em 15/02/2016.}

1 - Eng. Agrícola, Doutorando em Rec. Hídricos em Sistemas Agrícolas, DEG/UFLA, e-mail: caldasagri@yahoo.com.br

2 - Eng. Agrícola, Prof. Depto. de Engenharia, campus UFLA, Cep. 3037, Lavras, MG, e-mail: jacintoc@deg.ufla.br

3 - Eng. Agrícola, Depto. de Engenharia/UFLA, Campus Universitário, Lavras/MG, e-mail: frezende@deg.ufla.br 


\section{INTRODUÇÃO}

Água e energia elétrica são dois dos problemas básicos que irão influenciar, direta ou indiretamente, a vida de cerca de 200 milhões de brasileiros. Mesmo o Brasil detendo aproximadamente $12 \%$ de toda a água doce superficial do mundo, diversas regiões do país, recentemente, vêm sofrendo por desabastecimento de água, devido à combinação entre crescimento exagerado das demandas localizadas e da degradação da qualidade das águas (AZEVEDO et al., 2013), agravado por períodos prolongados de estiagem. Segundo Dong et al. (2013), problemas relacionados com a água estão atualmente perdendo apenas para questões energéticas, como ameaças para a sociedade humana. Apesar do potencial hidroelétrico brasileiro, foram observadas nas últimas décadas problemas de desabastecimento e racionamento, bem como o alto custo da energia que compromete a competitividade e o crescimento econômico do país (PIRES, 2008).

A agricultura, por meio da irrigação, é responsável pelo consumo de cerca de $80 \%$ de toda água destinada aos múltiplos usos no mundo, no Brasil esses valores chegam a $72 \%$. No entanto, em regiões com déficit hídrico, a irrigação assume papel primordial no desenvolvimento dos arranjos produtivos. Embora aumente o uso da água, os investimentos no setor resultam em aumento substancial da produtividade e do valor da produção, diminuindo a pressão pela incorporação de novas áreas para cultivo (ANA, 2015).Diante disso, ressalta-se a importância do uso da irrigação como acréscimo tecnológico de produção e a importância do uso racional da água e da energia elétrica no meio rural como forma de preservação desses recursos e redução dos custos de produção (SOUZA et al., 2005).

Diversos estudos são realizados visando maior eficiência nos projetos de adutoras para irrigação no Brasil, principalmente quando relacionados com a parte hidráulica (ZOCOLER et al., 2011). Mesmo que hidraulicamente correto, o projeto pode não atender satisfatoriamente aspectos econômicos (PERRONI et al., 2011). Portanto, no dimensionamento de adutoras são necessários estudos envolvendo variáveis hidráulicas e econômicas para a escolha adequada do diâmetro e do tipo de material da tubulação (ZOCOLER et al., 2004; DERCAS; VALIANTZAS, 2012).

Atualmente, diversos materiais estão disponíveis comercialmente para serem empregados na produção de tubos para adução e condução de água. Dentre os diversos materiais, os tubos de PVC e aço galvanizado são os mais utilizados por apresentarem diversas vantagens quando comparado aos outros materiais (FREIRE, 2000).

Diante dessa ampla gama de materiais, o projetista se vê em uma situação complexa de escolha, na tentativa de otimizar o uso, em função da falta de informações que o direcione para uma escolha mais eficiente, hidráulica e econômica. Baseado no exposto, objetivou-se com a realização do presente trabalho a obtenção de valores de velocidades de escoamento que induzem a um menor custo no dimensionamento da adutora em relação a vazão, o tempo de funcionamento do sistema, desnível geométrico, o comprimento da adutora, tarifas de energia elétrica e custo das tubulações de aço galvanizado e PVC e o período de funcionamento da adutora, considerando os custos atuais.

\section{MATERIAL E MÉTODOS}

Para realizar este estudo foi considerado um sistema de recalque padrão composto por uma válvula de pé com crivo, duas curvas de $90^{\circ}$ (raio curto), uma redução excêntrica e uma concêntrica, um registro de gaveta, uma curva dupla, uma válvula de retenção, uma bomba hidráulica com vazão máxima de $300 \mathrm{~m}^{3} \mathrm{~h}^{-1}$ e pressão máxima de $150 \mathrm{mca}$, acoplamento elástico, um motor elétrico trifásico, base de aço para conjunto motobomba, chave de partida e proteção e tubulações de aço galvanizado para pressão nominal de 180 mca e PVC para pressão 
nominal de 80,120 e 180 mca. Foram avaliadas vazões de 50,100,150, 200, 250 e $300 \mathrm{~m}^{3} \mathrm{~h}^{-1}$. Os preços dos componentes do sistema de recalque foram obtidos junto a diversos estabelecimentos comerciais de diferentes regiões do Brasil, afim de anular os efeitos dos impostos estaduais, sendo considerado o preço final ao consumidor no modo de pagamento à vista, no período de setembro a novembro de 2014.

O dimensionamento econômico foi obtido por meio da análise dos custos totais da adutora, composto pelos custos fixos e custos variáveis. Foi considerado como diâmetro econômico aquele que, para uma mesma situação, apresentou menor custo total.

Os custos fixos, aqueles que não variam de acordo com o volume de produção, foram definidos como sendo representados pelo custo de aquisição do sistema de recalque (custo da tubulação de sucção, motor elétrico, bomba hidráulica e tubulação), custo de assentamento dos tubos, custo de escavação e aterro de valas e a depreciação dos mesmos durante a sua vida útil.

Para determinação do custo das bombas hidráulicas centrífugas, foram coletados os preços de diferentes marcas e modelos existentes no mercado para atender às situações simuladas. Utilizando os catálogos técnicos fornecidos pelos fabricantes, foi selecionado, nas curvas características de cada bomba, o ponto de máximo rendimento e desse foram extraídos os valores de pressão e vazão. Adotou-se o ponto de máximo rendimento como sendo o ponto de operação da bomba.

$\mathrm{Na}$ determinação do custo dos tubos e acessórios, foram utilizados tubos de $6 \mathrm{~m}$ de comprimento de aço galvanizado flangeados e de PVC com ponta e bolsa e junta elástica. Para a tubulação de aço galvanizado, foram utilizados diâmetros de 75 a $500 \mathrm{~mm}$, com pressão nominal de 180 mca e, para a tubulação de PVC, foram utilizados diâmetros de 100 a $500 \mathrm{~mm}$, para pressão nominal de 80,125 e 180 mca. Os custos de assentamento dos tubos e de abertura e aterro de valas foram obtidos com base nos valores publicados pela Caixa Econômica Federal no Sistema Nacional de Pesquisa de Custos e Índices da Construção Civil (SINAPI, 2014), em tabela com referência para o mês de novembro de 2014.

Os custos variáveis, aqueles que variam com o volume de produção, foram definidos como sendo o somatório dos gastos com manutenção e energia elétrica. Os gastos com manutenção foram calculados de acordo com metodologia adotada por PRONI (1987), onde estes representam 2\% do custo fixo total.

Para determinação do custo da energia de bombeamento foram utilizadas diferentes situações de operação do sistema, envolvendo interações entre diferentes alturas manométricas, vazões, diâmetros, comprimentos de tubulação e tempos de funcionamento.

A altura manométrica foi determinada pelo somatório do desnível geométrico e a perda de carga total (contínua e localizada) nas tubulações de sucção e recalque, sendo que a mesma foi calculada utilizando a equação de HazenWilliams, adotando um coeficiente de rugosidade de 125 para tubulações de aço galvanizado e 140 para tubulações de PVC, ou seja, considerando como nova a tubulação. Foi utilizada a equação de Hazen-Williams por apresentar resultados consistentes para os materiais adotados e quando utilizada com diâmetros maiores que $50 \mathrm{~mm}$.

$\mathrm{O}$ consumo de energia pelos motores elétricos durante um ano foi obtido pelo produto entre a potência absorvida e o tempo de funcionamento do sistema de adução. Foram adotados tempos de funcionamento de 1000,2000 e $3000 \mathrm{~h} \mathrm{ano}^{-1}$.

A determinação do custo da energia elétrica foi baseada nos valores cobrados e critérios utilizados pela concessionária de energia elétrica do Estado de Minas Gerais (CEMIG), a qual divide os consumidores de acordo com a classificação da ANEEL, ou seja, por potência instalada: consumidores do grupo $\mathrm{B}$, com transformadores de até $75 \mathrm{kVA}$ e consumidores do grupo A, com transformadores maiores que 
$75 \mathrm{kVA}$.

Para o cálculo do custo de energia do grupo B, foi utilizada a seguinte Equação (1):

$\mathrm{CE}=\mathrm{C}_{\mathrm{ee}} \cdot \mathrm{T}_{\mathrm{e}} \cdot \mathrm{T}_{\mathrm{f}}$

em que,

$\mathrm{CE}=$ custo com energia, $\mathrm{R} \$$;

$\mathrm{C}_{\mathrm{ee}}=$ consumo da energia elétrica, $\mathrm{kWh}$;

$\mathrm{T}_{\mathrm{e}}=$ tarifa de energia elétrica, $\mathrm{R} \$ \mathrm{kWh}^{-1}$; e

$\mathrm{T}_{\mathrm{f}}=$ tempo de funcionamento, $\mathrm{h} \mathrm{ano}^{-1}$.

Para o cálculo do custo de energia dos consumidores do grupo A, foi considerado o consumo de energia $(\mathrm{kWh})$ e a demanda de potência $(\mathrm{kW})$. Foram utilizados o sistema de tarifação modalidade convencional, que não diferencia a tarifa conforme o período do ano e a hora do dia, e os sistemas de tarifação horosazonal Verde e Azul, que fazem distinção conforme o período do ano e a hora do dia. Foram simuladas situações, para o período de funcionamento fora de ponta seco (FPS), período de ponta seco (PS), fora de ponta úmido (FPU) e na ponta úmido (PU). O custo da energia anual para os consumidores do grupo A, foi obtida pela Equação (2) para modalidade convencional e pela Equação (3) para Modalidade horosazonal Verde e horosazonal Azul:

$C E=12 \cdot D_{c} \cdot T_{d}+C_{e e} \cdot T_{e} \cdot T_{f}$

em que,

$\mathrm{CE}=$ custo com energia, $\mathrm{R} \$$ ano $^{-1}$;

$\mathrm{D}_{\mathrm{c}}=$ demanda contratada, $\mathrm{kW}$;

$\mathrm{T}_{\mathrm{d}}=$ tarifa de demanda, $\mathrm{R} \$ \mathrm{~kW}^{-1}$;

$\mathrm{C}_{\mathrm{ee}}=$ consumo de energia elétrica, $\mathrm{kWh}$;

$\mathrm{T}_{\mathrm{e}}=$ tarifa de consumo (modalidade convencional), $\mathrm{R} \$ \mathrm{kWh}^{-1}$;

$\mathrm{T}_{\mathrm{f}}=$ tempo de funcionamento, $\mathrm{h}$ ano ${ }^{-1}$.

Foram considerados os valores de tarifação aplicados pela CEMIG, divulgados para o mês de novembro de 2014, sendo simuladas situações em que o valor de demanda não ultrapasse a demanda contratada, evitando multas. Foram considerados acréscimos de 30 e $100 \%$ nos custos atuais de energia de bombeamento e os mesmos valores de acréscimos nos custos atuais de aquisição dos tubos e acessórios.

$C E=12 \cdot D_{c} \cdot T_{d}+C_{e f p s} \cdot T_{e f p s} \cdot T_{f f p s}+C_{e f p u} \cdot T_{\text {efpu }} \cdot T_{\text {ffpu }}+C_{\text {epu }} \cdot T_{\text {epu }} \cdot T_{\text {fpu }}+C_{\text {eps }} \cdot T_{\text {eps }} \cdot T_{\text {fps }}$

em que,

$\mathrm{CE}=$ custo com energia, $\mathrm{R} \$ \mathrm{ano}^{-1}$;

$\mathrm{D}_{\mathrm{c}}=$ demanda contratada, $\mathrm{kW}$;

$\mathrm{T}_{\mathrm{d}}=$ tarifa de demanda, $\mathrm{R} \$ \mathrm{~kW}^{-1}$;

$\mathrm{C}_{\text {efps }}=$ consumo de energia no período fora de ponta seco, $\mathrm{kWh}$;

$\mathrm{T}_{\text {efps }}=$ tarifa de consumo no período fora de ponta seco, $\mathrm{R} \$ \mathrm{kWh}^{-1}$;

$\mathrm{T}_{\text {ffps }}=$ tempo de funcionamento no período fora de ponta seco, $\mathrm{h} \mathrm{ano}{ }^{-1}$;

$\mathrm{C}_{\text {efpu }}=$ consumo de energia no período fora de ponta úmido, $\mathrm{kWh}$;

$\mathrm{T}_{\text {efup }}=$ tarifa de consumo no período fora de ponta úmido, $\mathrm{R} \$ \mathrm{kWh}^{-1}$;

$\mathrm{T}_{\text {ffpu }}=$ tempo de funcionamento no período fora de ponta úmido, $\mathrm{h} \mathrm{ano}^{-1}$.

$\mathrm{C}_{\mathrm{epu}}=$ consumo de energia no período de ponta úmido, $\mathrm{kWh}$;

$\mathrm{T}_{\mathrm{epu}}=$ tarifa de consumo no período de ponta úmido, $\mathrm{R} \$ \mathrm{kWh}^{-1}$;

$\mathrm{T}_{\mathrm{fpu}}=$ tempo de funcionamento no período de ponta úmido, $\mathrm{h}$ ano ${ }^{-1}$;

$\mathrm{C}_{\text {eps }}=$ consumo de energia no período de ponta seco, $\mathrm{kWh}$;

$\mathrm{T}_{\mathrm{eps}}=$ tarifa de consumo no período de ponta seco, $\mathrm{R} \$ \mathrm{kWh}^{-1} ; \mathrm{e}$

$\mathrm{T}_{\mathrm{fps}}=$ tempo de funcionamento no período de ponta seco, $\mathrm{h}_{\mathrm{ano}}^{-1}$. 


\section{RESULTADOS E DISCUSSÕES}

Nos Quadros 1 e 2 são apresentados os resultados de velocidade econômica para tubulação de aço galvanizado, considerando desnível geométrico total de 50 e $150 \mathrm{~m}$, respectivamente, para tempos de funcionamento 1000,2000 e $3000 \mathrm{~h} \mathrm{ano}^{-1}$, e diferentes tarifas energéticas.

Comparando os resultados obtidos nos Quadros 1 e 2, para adutoras de aço galvanizado, nota-se que não houve diferença de valores de velocidade econômica para as vazões de 50 e $250 \mathrm{~m}^{3} \mathrm{~h}^{-1} \mathrm{em}$ função do aumento no desnível geométrico total. Para a vazão de $100 \mathrm{~m}^{3} \mathrm{~h}^{-1}$ houve diminuição da velocidade econômica, nos tempos de funcionamento de 1000 e $2000 \mathrm{~h}^{a n o}{ }^{-1}$. Para 150 $\mathrm{m}^{3} \mathrm{~h}^{-1}$ houve redução da velocidade no tempo de funcionamento de $1000 \mathrm{~h} \mathrm{ano}^{-1} \mathrm{em}$ todos os sistemas tarifários analisados: Convencional, Verde e Azul. Para $200 \mathrm{~m}^{3} \mathrm{~h}^{-1}$, houve aumento da velocidade para o tempo de funcionamento de $1000 \mathrm{~h} \mathrm{ano}^{-1}$ em todos os sistemas tarifários. Para a vazão 300 $\mathrm{m}^{3} \mathrm{~h}^{-1}$ houve aumento na velocidade para todos os tempos de $1000 \mathrm{~h}_{\text {ano }}^{-1} \mathrm{e}$ no sistema tarifário Verde, houve aumento da velocidade econômica para o tempo de 2000 horas ano ${ }^{-1}$. Para os demais tempos de funcionamento, houve redução da velocidade econômica. Pode-se verificar também que, a partir da vazão de $150 \mathrm{~m}^{3} \mathrm{~h}^{-1}$, com o aumento do desnível geométrico, houve mudança no grupo de tarifa de

Quadro 1. Velocidade econômica $\left(\mathrm{m} \mathrm{s}^{-1}\right)$ para tubulação de aço galvanizado com tempo de funcionamento de 1000,2000 e $3000 \mathrm{~h}_{\text {ano }}{ }^{-1}$, considerando diferentes tarifas energéticas (Convencional, Verde e Azul) e desnível geométrico total de $50 \mathrm{~m}$.

\begin{tabular}{|c|c|c|c|c|c|c|c|c|c|}
\hline \multicolumn{10}{|c|}{$50 \mathrm{~m}$} \\
\hline \multirow{2}{*}{$\begin{array}{l}\text { Vazão } \\
\left(\mathrm{m}^{3} \mathrm{~h}^{-1}\right)\end{array}$} & \multicolumn{3}{|c|}{ Convencional } & \multicolumn{3}{|c|}{ Verde } & \multicolumn{3}{|c|}{ Azul } \\
\hline & $1000 \mathrm{~h}$ & $2000 \mathrm{~h}$ & $3000 \mathrm{~h}$ & $1000 \mathrm{~h}$ & $2000 \mathrm{~h}$ & $3000 \mathrm{~h}$ & $1000 \mathrm{~h}$ & $2000 \mathrm{~h}$ & $3000 \mathrm{~h}$ \\
\hline 50 & 1,76 & 1,13 & 1,13 & 1,76 & 1,13 & 1,13 & 1,76 & 1,13 & 1,13 \\
\hline 100 & 2,26 & 2,26 & 1,57 & 2,26 & 2,26 & 1,57 & 2,26 & 2,26 & 1,57 \\
\hline 150 & 2,35 & 1,72 & 1,32 & 2,35 & 1,32 & 1,32 & 2,35 & 1,72 & 1,32 \\
\hline 200 & 1,76 & 1,76 & 1,76 & 1,76 & 1,76 & 1,76 & 1,76 & 1,76 & 1,76 \\
\hline 250 & 2,21 & 2,21 & 2,21 & 2,21 & 2,21 & 2,21 & 2,21 & 2,21 & 2,21 \\
\hline 300 & 1,70 & 1,70 & 1,70 & 1,70 & 1,70 & 1,70 & 1,70 & 1,70 & 1,70 \\
\hline
\end{tabular}

*Valores de velocidade média sombreados correspondem à tarifa de cobrança do grupo A, valores não sombreados grupo $\mathrm{B}$.

Quadro 2. Velocidade econômica $\left(\mathrm{m} \mathrm{s}^{-1}\right)$ para tubulação de aço galvanizado com tempo de funcionamento de 1000,2000 e $3000 \mathrm{~h} \mathrm{ano}^{-1}$, considerando diferentes tarifas energéticas (Convencional, Verde e Azul) e desnível geométrico total de $150 \mathrm{~m}$.

\begin{tabular}{cccccccccccc}
\hline \multicolumn{10}{c}{$150 \mathrm{~m}$} \\
\hline \multirow{2}{*}{ Vazão $\left(\mathrm{m}^{3} \mathrm{~h}^{-1}\right)$} & \multicolumn{9}{c}{ Convencional } & \multicolumn{1}{c}{ Verde } & \multicolumn{3}{c}{ Azul } \\
\cline { 2 - 13 } & $1000 \mathrm{~h}$ & $2000 \mathrm{~h}$ & $3000 \mathrm{~h}$ & $1000 \mathrm{~h}$ & $2000 \mathrm{~h}$ & $3000 \mathrm{~h}$ & $1000 \mathrm{~h}$ & $2000 \mathrm{~h}$ & $3000 \mathrm{~h}$ \\
\hline 50 & 1,76 & 1,13 & 1,13 & 1,76 & 1,13 & 1,13 & 1,76 & 1,13 & 1,13 \\
100 & 1,57 & 1,57 & 1,57 & 1,57 & 1,57 & 2,26 & 1,57 & 1,57 & 2,26 \\
150 & 1,72 & 1,72 & 1,32 & 1,72 & 1,72 & 1,32 & 1,72 & 1,72 & 1,32 \\
200 & 2,30 & 1,76 & 1,76 & 2,30 & 1,76 & 1,76 & 2,30 & 1,76 & 1,76 \\
250 & 2,21 & 2,21 & 2,21 & 2,21 & 2,21 & 2,21 & 2,21 & 2,21 & 2,21 \\
300 & 2,65 & 1,17 & 1,17 & 2,65 & 2,65 & 1,17 & 1,70 & 2,65 & 1,17 \\
\hline
\end{tabular}

*Valores de velocidade média sombreados correspondem à tarifa de cobrança do grupo A, valores não sombreados grupo B. 
cobrança energética, passando do grupo B para o grupo A.

Esses resultados indicam que, aumentando o desnível geométrico total, ocorre um aumento na demanda de potência necessária para acionamento da adutora, que elevam os gastos com energia elétrica, consequentemente os custos variáveis; sendo necessário um acréscimo no diâmetro da tubulação para equilibrar os custos, que por sua vez ocasionam em menores valores de velocidades de escoamento para uma mesma vazão.

Gomes \& Silva (2006) e Lima et al. (2009) citam que a redução no diâmetro da tubulação eleva as perdas de carga e aumenta a potência necessária do conjunto motobomba; esta configuração de projeto propicia maior custo do conjunto elevatório e maior despesa com energia, embora proporcione economia na compra da tubulação. Por outro lado, aumentando-se o diâmetro da tubulação, a potência do conjunto elevatório e o custo operacional de energia elétrica serão menores, podendo tornar-se economicamente viável ao longo da vida útil dos equipamentos.

Similarmente como para as tubulações de aço galvanizado acima, nos Quadros 3 e 4 são apresentados os resultados de velocidade econômica para tubulação de PVC.

Os resultados apresentados nos Quadros 4

Quadro 3. Velocidade econômica $\left(\mathrm{m} \mathrm{s}^{-1}\right)$ para tubulação de PVC com tempo de funcionamento de 1000, 2000 e $3000 \mathrm{~h} \mathrm{ano}^{-1}$, considerando diferentes tarifas energéticas (Convencional, Verde e Azul) e desnível geométrico total de $50 \mathrm{~m}$.

\begin{tabular}{cccccccccccc}
\hline \multicolumn{10}{c}{$50 \mathrm{~m}$} \\
\hline \multirow{2}{*}{ Vazão $\left(\mathrm{m}^{3} \mathrm{~h}^{-1}\right)$} & \multicolumn{9}{c}{ Convencional } & \multicolumn{1}{c}{ Verde } & \multicolumn{3}{c}{ Azul } \\
\cline { 2 - 10 } & $1000 \mathrm{~h}$ & $2000 \mathrm{~h}$ & $3000 \mathrm{~h}$ & $1000 \mathrm{~h}$ & $2000 \mathrm{~h}$ & $3000 \mathrm{~h}$ & $1000 \mathrm{~h}$ & $2000 \mathrm{~h}$ & $3000 \mathrm{~h}$ \\
\hline 50 & 1,41 & 1,41 & 1,41 & 1,41 & 1,41 & 1,41 & 1,41 & 1,41 & 1,41 \\
100 & 1,36 & 1,36 & 1,36 & 1,36 & 1,36 & 1,36 & 1,36 & 1,36 & 1,36 \\
150 & 1,23 & 1,23 & 1,23 & 1,23 & 1,23 & 1,23 & 1,23 & 1,23 & 1,23 \\
200 & 1,64 & 1,64 & 1,64 & 1,64 & 1,64 & 1,64 & 1,64 & 1,64 & 1,64 \\
250 & 2,05 & 2,05 & 1,34 & 2,05 & 2,05 & 1,34 & 2,05 & 2,05 & 1,34 \\
300 & 1,61 & 1,61 & 1,61 & 1,61 & 1,61 & 1,61 & 1,61 & 1,61 & 1,61 \\
\hline
\end{tabular}

*Valores de velocidade média sombreados correspondem à tarifa de cobrança do grupo A, valores não sombreados grupo B.

Quadro 4. Velocidade econômica $\left(\mathrm{m} \mathrm{s}^{-1}\right)$ para tubulação de PVC com tempo de funcionamento de 1000, 2000 e $3000 \mathrm{~h} \mathrm{ano}^{-1}$, considerando diferentes tarifas energéticas (Convencional, Verde e Azul) e desnível geométrico total de $150 \mathrm{~m}$.

\begin{tabular}{|c|c|c|c|c|c|c|c|c|c|}
\hline \multicolumn{10}{|c|}{$150 \mathrm{~m}$} \\
\hline \multirow{2}{*}{ Vazão $\left(m^{3} h^{-1}\right)$} & \multicolumn{3}{|c|}{ Convencional } & \multicolumn{3}{|l|}{ Verde } & \multicolumn{3}{|l|}{ Azul } \\
\hline & $1000 \mathrm{~h}$ & $2000 \mathrm{~h}$ & $3000 \mathrm{~h}$ & $1000 \mathrm{~h}$ & $2000 \mathrm{~h}$ & $3000 \mathrm{~h}$ & $1000 \mathrm{~h}$ & $2000 \mathrm{~h}$ & $3000 \mathrm{~h}$ \\
\hline 50 & 1,42 & 1,42 & 1,42 & 1,42 & 1,42 & 1,42 & 1,42 & 1,42 & 1,42 \\
\hline 100 & 1,37 & 1,37 & 1,37 & 1,37 & 1,37 & 1,37 & 1,37 & 1,37 & 1,37 \\
\hline 150 & 1,21 & 1,21 & 1,21 & 1,21 & 1,21 & 1,21 & 1,21 & 1,21 & 1,21 \\
\hline 200 & 1,61 & 1,61 & 1,61 & 1,61 & 1,61 & 1,61 & 1,61 & 1,61 & 1,61 \\
\hline 250 & 2,01 & 2,01 & 2,01 & 2,01 & 2,01 & 2,01 & 2,01 & 2,01 & 2,01 \\
\hline 300 & 1,58 & 1,58 & 1,58 & 1,58 & 1,58 & 1,58 & 1,58 & 1,58 & 1,58 \\
\hline
\end{tabular}

*Valores de velocidade média sombreados correspondem à tarifa de cobrança do grupo A, valores não sombreados grupo $\mathrm{B}$. 
e 5 indicam que a velocidade econômica nas tubulações de PVC não variou em função do aumento do desnível geométrico, sendo este responsável somente pela alteração do grupo de tarifa energética, passando do grupo B para o grupo $\mathrm{A}$, a partir da vazão de $150 \mathrm{~m}^{3} \mathrm{~h}^{-1}$. Os valores próximos de velocidades econômicas obtidos são devido a diferenças no diâmetro interno dos tubos utilizados para atender as diversas situações de simuladas. No caso do Quadro 4, foi utilizado o tubo PN80 e no Quadro 5, o PN180. O fato das velocidades econômicas não terem variados pode ser explicado devido à tubulação de PVC apresentar, quando comparado com os tubos de aço galvanizado, menor coeficiente de rugosidade, o que sugere que a demanda de potência ocorrida na simulação não foi suficiente para causar alteração no diâmetro econômico. Resultado semelhante foi obtido por Carvalho e Reis (2000), considerando alturas geométricas de 30 e $60 \mathrm{~m}$, sendo que os valores de velocidade econômica não apresentaram comportamento definido em relação ao desnível geométrico total.

Nos Quadros 5 e 6 estão apresentados os resultados de velocidade econômica para tubulação de aço galvanizado, considerando acréscimo de $30 \%$ e $100 \%$, respectivamente, nos custos atuais de energia de bombeamento, para tempos de funcionamento de 1000,2000 e $3000 \mathrm{~h}^{2} \mathrm{no}^{-1} \mathrm{e}$ diferentes tarifas energéticas.

Quadro 5. Velocidade econômica $\left(\mathrm{m} \mathrm{s}^{-1}\right)$ para a tubulação de aço galvanizado com tempo de funcionamento de 1000, 2000 e $3000 \mathrm{~h}_{\text {ano }}^{-1}$ considerando diferentes tarifas energéticas (Convencional, Verde e Azul), 30\% de aumento nos custos atuais de energia de bombeamento e desnível geométrico total de $50 \mathrm{~m}$.

\begin{tabular}{ccccccccccc}
\hline \multicolumn{10}{c}{$30 \%$} \\
\hline \multirow{2}{*}{ Vazão $\left(\mathrm{m}^{3} \mathrm{~h}^{-1}\right)$} & \multicolumn{9}{c}{ Convencional } & \multicolumn{3}{c}{ Verde } & \multicolumn{3}{c}{ Azul } \\
\cline { 2 - 12 } & $1000 \mathrm{~h}$ & $2000 \mathrm{~h}$ & $3000 \mathrm{~h}$ & $1000 \mathrm{~h}$ & $2000 \mathrm{~h}$ & $3000 \mathrm{~h}$ & $1000 \mathrm{~h}$ & $2000 \mathrm{~h}$ & $3000 \mathrm{~h}$ \\
\hline 50 & 1,13 & 1,13 & 1,13 & 1,13 & 1,13 & 1,13 & 1,13 & 1,13 & 1,13 \\
100 & 2,26 & 1,57 & 1,15 & 2,26 & 1,57 & 1,15 & 2,26 & 1,57 & 1,15 \\
150 & 1,72 & 1,32 & 1,32 & 1,72 & 1,32 & 1,32 & 1,72 & 1,32 & 1,32 \\
200 & 1,76 & 1,76 & 1,76 & 1,76 & 1,76 & 1,76 & 1,76 & 1,76 & 1,76 \\
250 & 2,21 & 2,21 & 1,41 & 2,21 & 2,21 & 1,41 & 2,21 & 2,21 & 1,41 \\
300 & 1,70 & 1,70 & 1,17 & 1,70 & 1,70 & 1,17 & 1,70 & 1,70 & 1,17 \\
\hline
\end{tabular}

*Valores de velocidade média sombreados correspondem à tarifa de cobrança do grupo A, valores não sombreados grupo B.

Quadro 6. Velocidade econômica $\left(\mathrm{m} \mathrm{s}^{-1}\right)$ para a tubulação de aço galvanizado com tempo de funcionamento de 1000, 2000 e $3000 \mathrm{~h} \mathrm{ano}^{-1}$ considerando diferentes tarifas energéticas (Convencional, Verde e Azul), $100 \%$ de aumento nos custos atuais de energia de bombeamento e desnível geométrico total de $50 \mathrm{~m}$.

\begin{tabular}{|c|c|c|c|c|c|c|c|c|c|}
\hline \multirow{3}{*}{ Vazão $\left(\mathrm{m}^{3} \mathrm{~h}^{-1}\right)$} & \multicolumn{6}{|c|}{$100 \%$} & \multirow{2}{*}{\multicolumn{3}{|c|}{ Azul }} \\
\hline & \multicolumn{3}{|c|}{ Convencional } & \multicolumn{3}{|c|}{ Verde } & & & \\
\hline & $1000 \mathrm{~h}$ & $2000 \mathrm{~h}$ & $3000 \mathrm{~h}$ & $1000 \mathrm{~h}$ & $2000 \mathrm{~h}$ & $3000 \mathrm{~h}$ & $1000 \mathrm{~h}$ & $2000 \mathrm{~h}$ & $3000 \mathrm{~h}$ \\
\hline 50 & 1,13 & 1,13 & 1,13 & 1,13 & 1,13 & 1,13 & 1,13 & 1,13 & 1,13 \\
\hline 100 & 1,57 & 1,15 & 1,15 & 1,57 & 1,15 & 1,15 & 1,57 & 1,15 & 1,15 \\
\hline 150 & 1,32 & 1,32 & 1,32 & 1,32 & 1,32 & 1,32 & 1,32 & 1,32 & 1,32 \\
\hline 200 & 1,76 & 1,76 & 1,13 & 1,76 & 1,76 & 1,13 & 1,76 & 1,76 & 1,13 \\
\hline 250 & 2,21 & 1,41 & 0,98 & 2,21 & 1,41 & 0,98 & 2,21 & 1,41 & 0,98 \\
\hline 300 & 1,70 & 1,17 & 1,17 & 1,70 & 1,17 & 1,17 & 1,70 & 1,17 & 1,17 \\
\hline
\end{tabular}

*Valores de velocidade média sombreados correspondem à tarifa de cobrança do grupo A, valores não sombreados grupo B. 
Comparando os resultados obtidos nos Quadros 5 e 6 , nota-se que não houve alteração da velocidade econômica de escoamento para a vazão de $50 \mathrm{~m}^{3} \mathrm{~h}^{-1}$ em função do aumento nos custos de energia. Para $100 \mathrm{~m}^{3} \mathrm{~h}^{-1}$ houve diminuição da velocidade nos tempos de funcionamento de $1000 \mathrm{e} 2000 \mathrm{~h} \mathrm{ano}^{-1}$, e a mesma permaneceu constante para $3000 \mathrm{~h} \mathrm{ano}^{-1}$. Houve diminuição da velocidade para a vazão de $150 \mathrm{~m}^{3} \mathrm{~h}^{-1}$, no tempo de funcionamento de 1000 $\mathrm{h}$ ano ${ }^{-1}$, para os demais tempos de funcionamento $\left(2000\right.$ e $\left.3000 \mathrm{~h}^{2} \mathrm{ano}^{-1}\right)$ a velocidade permaneceu constante. Para a vazão de $200 \mathrm{~m}^{3} \mathrm{~h}^{-1}$, não houve variação da velocidade, para os tempos de funcionamento de 1000 e $2000 \mathrm{~h}^{2} \mathrm{no}^{-1}$, no entanto, com o aumento do tempo de funcionamento para $3000 \mathrm{~h} \mathrm{ano}^{-1}$, houve diminuição da velocidade econômica. Para a vazão de $250 \mathrm{~m}^{3} \mathrm{~h}^{-1}$, para o tempo de funcionamento de $1000 \mathrm{~h} \mathrm{ano}^{-1} \mathrm{a}$ velocidade de escoamento permaneceu constante, para os demais tempos houve redução da velocidade. Para a vazão de $300 \mathrm{~m}^{3} \mathrm{~h}^{-1}$, nos tempos de funcionamento de 1000 e $3000 \mathrm{~h} \mathrm{ano}^{-1}$, a velocidade permaneceu constante e, para o tempo de funcionamento de $2000 \mathrm{~h}^{2} \mathrm{no}^{-1}$, a velocidade reduziu.

Analisando os resultados, percebe-se que a diminuição da velocidade econômica obtida em algumas situações, entre os comparativos acréscimos de $30 \%$ para $100 \%$, sobre os custos atuais de tarifa energética, pode ser justificada devido à necessidade do uso de diâmetros maiores, para uma mesma vazão, no intuito de reduzir os custos variáveis, ou seja, aqueles advindos principalmente da perda de carga na tubulação, consequentemente reduzir os gastos com energia de bombeamento.

Comparando os resultados obtidos neste trabalho com o de Perroni (2008), que analisou a influência do aumento da tarifa energética sobre a velocidade econômica, para diferentes estados brasileiros (Minas Gerais, São Paulo, Rio de Janeiro, Paraná e Goiás), verifica-se resultado semelhante, sendo que o autor ainda cita que maiores valores de gasto com energia elétrica acarretam em elevação dos custos totais através dos custos variáveis, sendo necessário reduzir a potência necessária ao funcionamento do sistema a qual só pode ser proveniente de uma redução do desnível geométrico total.
Perroni et al. (2015), estudando adutoras de aço galvanizado, compararam o efeito do aumento do custo da energia elétrica em função do comprimento da tubulação, sobre a velocidade econômica e concluíram que adutoras de pequena extensão (menores que $100 \mathrm{~m}$ ) são mais sensíveis ao aumento da energia. De acordo com os autores, em adutoras de aço galvanizado de menor extensão, o valor inicial de investimento com a aquisição da tubulação tem menor representatividade no custo total do sistema, assim o aumento da energia tem maior impacto nos custos finais, resultando em diâmetro econômico maior e, consequentemente, velocidade econômica menor.

Nos Quadros 7 e 8 estão os resultados de velocidade econômica para tubulação de PVC, considerando acréscimo de $30 \%$ e $100 \%$, respectivamente, nos custos atuais de energia de bombeamento, permanecendo os custos atuais de aquisição da tubulação, para tempos de funcionamento de 1000,2000 e $3000 \mathrm{~h} \mathrm{ano}^{-1}$ e diferentes tarifas energéticas.

Os resultados apresentados nos Quadros 8 e 9 indicam que, para as vazões de 150 e $250 \mathrm{~m}^{3} \mathrm{~h}^{-1}$, a velocidade econômica não variou em função do acréscimo dado na tarifa de energia. Para a vazão de $50 \mathrm{~m}^{3} \mathrm{~h}^{-1}$ houve redução da velocidade para os tempos de funcionamento de 2000 e $3000 \mathrm{~h}$ ano $^{-1}$, no entanto, para $1000 \mathrm{~h} \mathrm{ano}^{-1}$, a velocidade permaneceu constante. Para $100 \mathrm{~m}^{3} \mathrm{~h}^{-1}$, a velocidade reduziu apenas para o tempo de funcionamento de $2000 \mathrm{~h} \mathrm{ano}^{-1}$, permanecendo constante para os demais tempos de funcionamento. Na vazão de $200 \mathrm{~m}^{3} \mathrm{~h}^{-1}$, houve variação, com redução da vazão, apenas para o tempo de funcionamento de $3000 \mathrm{~h}$ ano ${ }^{-1}$. Para a vazão de $300 \mathrm{~m}^{3} \mathrm{~h}^{-1}$, houve variação apenas para o tempo de funcionamento de $3000 \mathrm{~h}$ ano $^{-1}$, nos sistemas de tarifação Verde e Azul.

Assim como nos resultados obtidos para a tubulação de aço galvanizado, a diminuição da velocidade econômica para a tubulação de PVC pode ser explicada devido ao fato de que para uma mesma vazão é necessário diâmetros maiores para reduzir os gastos com energia de bombeamentos oriundos da perda de carga, vistos os aumentos simulados de $30 \%$ e $100 \%$ sobre os custos atuais. Ou seja, o custo da energia elétrica é parte integrante do custo final e sua interferência nas

\section{REVENG}


Quadro 7. Velocidade econômica $\left(\mathrm{m} \mathrm{s}^{-1}\right)$ para a tubulação de PVC com tempo de funcionamento de 1000 , 2000 e $3000 \mathrm{~h} \mathrm{ano}^{-1}$ considerando diferentes tarifas energéticas (Convencional, Verde e Azul), $30 \%$ de aumento nos custos atuais de energia de bombeamento e desnível geométrico total de $50 \mathrm{~m}$.

\begin{tabular}{ccccccccccc}
\hline \multicolumn{10}{c}{$30 \%$} \\
\hline \multirow{2}{*}{ Vazão $\left(\mathrm{m}^{3} \mathrm{~h}^{-1}\right)$} & \multicolumn{9}{c}{ Convencional } & \multicolumn{1}{c}{ Verde } \\
\cline { 2 - 12 } & $1000 \mathrm{~h}$ & $2000 \mathrm{~h}$ & $3000 \mathrm{~h}$ & $1000 \mathrm{~h}$ & $2000 \mathrm{~h}$ & $3000 \mathrm{~h}$ & $1000 \mathrm{~h}$ & $2000 \mathrm{~h}$ & $3000 \mathrm{~h}$ \\
\hline 50 & 1,41 & 1,41 & 0,68 & 1,41 & 1,41 & 0,68 & 1,41 & 1,41 & 0,68 \\
100 & 1,36 & 1,36 & 0,82 & 1,36 & 1,36 & 0,82 & 1,36 & 1,36 & 0,82 \\
150 & 1,23 & 1,23 & 1,23 & 1,23 & 1,23 & 1,23 & 1,23 & 1,23 & 1,23 \\
200 & 1,64 & 1,64 & 1,64 & 1,64 & 1,64 & 1,64 & 1,64 & 1,64 & 1,64 \\
250 & 2,05 & 1,34 & 1,34 & 2,05 & 1,34 & 1,34 & 2,05 & 1,34 & 1,34 \\
300 & 1,61 & 1,61 & 1,61 & 1,61 & 1,61 & 1,61 & 1,61 & 1,61 & 1,61 \\
\hline
\end{tabular}

*Valores de velocidade média sombreados correspondem à tarifa de cobrança do grupo A, valores não sombreados grupo $\mathrm{B}$.

Quadro 8. Velocidade econômica $\left(\mathrm{m} \mathrm{s}^{-1}\right)$ para a tubulação de PVC com tempo de funcionamento de 1000, 2000 e $3000 \mathrm{~h} \mathrm{ano}^{-1}$ considerando diferentes tarifas energéticas (Convencional, Verde e Azul), $100 \%$ de aumento nos custos atuais de energia de bombeamento e desnível geométrico total de $50 \mathrm{~m}$.

\begin{tabular}{cccccccccccc}
\hline \multicolumn{10}{c}{$100 \%$} \\
\hline \multirow{2}{*}{ Vazão $\left(\mathrm{m}^{3} \mathrm{~h}^{-1}\right)$} & \multicolumn{9}{c}{ Convencional } & \multicolumn{1}{c}{ Verde } & \multicolumn{3}{c}{ Azul } \\
\cline { 2 - 12 } & $1000 \mathrm{~h}$ & $2000 \mathrm{~h}$ & $3000 \mathrm{~h}$ & $1000 \mathrm{~h}$ & $2000 \mathrm{~h}$ & $3000 \mathrm{~h}$ & $1000 \mathrm{~h}$ & $2000 \mathrm{~h}$ & $3000 \mathrm{~h}$ \\
\hline 50 & 1,41 & 0,68 & 0,68 & 1,41 & 0,68 & 0,68 & 1,41 & 0,68 & 0,68 \\
100 & 1,36 & 0,82 & 0,82 & 1,36 & 0,82 & 0,82 & 1,36 & 0,82 & 0,82 \\
150 & 1,23 & 1,23 & 1,23 & 1,23 & 1,23 & 1,23 & 1,23 & 1,23 & 1,23 \\
200 & 1,64 & 1,64 & 1,07 & 1,64 & 1,64 & 1,07 & 1,64 & 1,64 & 1,07 \\
250 & 2,05 & 1,34 & 1,34 & 2,05 & 1,34 & 1,34 & 2,05 & 1,34 & 1,34 \\
300 & 1,61 & 1,61 & 1,61 & 1,61 & 1,61 & 1,13 & 1,61 & 1,61 & 1,13 \\
\hline
\end{tabular}

*Valores de velocidade média sombreados correspondem à tarifa de cobrança do grupo A, valores não sombreados grupo B.

variáveis de otimização é direta, como mencionado por Marcuzzo \& Wendland (2010).

De acordo com Carvalho e Reis (2000), em relação ao tempo de funcionamento, quanto maior ele for maior será o gasto com energia, ou seja, aumentam os custos finais. Como resultado, o valor da velocidade econômica diminui à medida que aumenta o tempo de funcionamento.A análise dos gastos com energia de bombeamento é de suma importância no dimensionamento econômico de uma adutora, visto que dentro dos custos variáveis a energia de bombeamento pode chegar a representar, dependendo do método de irrigação, a
70\% desses custos (MELO, 1993).

Nos Quadros 9 e 10 estão apresentados os resultados de velocidade econômica para a tubulação de aço galvanizado considerando um acréscimo de 30 e $100 \%$, respectivamente, nos custos atuais de aquisição da tubulação, permanecendo os custos atuais de energia de bombeamento, tempo de funcionamento de 1000 , 2000 e $3000 \mathrm{~h} \mathrm{ano}^{-1}$ e diferentes tarifas energéticas.

O aumento no custo da tubulação, como os observados nos Quadros 9 e 10, provocou um aumento na velocidade econômica para a vazão de $50 \mathrm{~m}^{3} \mathrm{~h}^{-1}$, no tempo de funcionamento de 2000 
Quadro 9. Velocidade econômica $\left(\mathrm{m} \mathrm{s}^{-1}\right)$ para a tubulação de aço galvanizado com tempo de funcionamento de 1000,2000 e $3000 \mathrm{~h} \mathrm{ano}^{-1}$ considerando diferentes tarifas energéticas (Convencional, Verde e Azul), 30\% de aumento nos custos atuais de aquisição da tubulação e desnível geométrico total de $50 \mathrm{~m}$.

\begin{tabular}{|c|c|c|c|c|c|c|c|c|c|}
\hline \multirow{3}{*}{ Vazão $\left(\mathrm{m}^{3} \mathrm{~h}^{-1}\right)$} & \multicolumn{6}{|c|}{$30 \%$} & & & \\
\hline & \multicolumn{3}{|c|}{ Convencional } & \multicolumn{3}{|c|}{ Verde } & \multicolumn{3}{|c|}{ Azul } \\
\hline & $1000 \mathrm{~h}$ & $2000 \mathrm{~h}$ & $3000 \mathrm{~h}$ & $1000 \mathrm{~h}$ & $2000 \mathrm{~h}$ & $3000 \mathrm{~h}$ & $1000 \mathrm{~h}$ & $2000 \mathrm{~h}$ & $3000 \mathrm{~h}$ \\
\hline 50 & 1,76 & 1,13 & 1,13 & 1,76 & 1,13 & 1,13 & 1,76 & 1,13 & 1,13 \\
\hline 100 & 2,26 & 2,26 & 1,57 & 2,26 & 2,26 & 1,57 & 2,26 & 2,26 & 1,57 \\
\hline 150 & 2,35 & 1,72 & 1,32 & 2,35 & 1,72 & 1,32 & 2,35 & 1,72 & 1,32 \\
\hline 200 & 1,76 & 1,76 & 1,76 & 1,76 & 1,76 & 1,76 & 1,76 & 1,76 & 1,76 \\
\hline 250 & 2,21 & 2,21 & 2,21 & 2,21 & 2,21 & 2,21 & 2,21 & 2,21 & 2,21 \\
\hline 300 & 1,70 & 1,70 & 1,70 & 2,65 & 1,70 & 1,70 & 2,65 & 1,70 & 1,70 \\
\hline
\end{tabular}

*Valores de velocidade média sombreados correspondem à tarifa de cobrança do grupo A, valores não sombreados grupo $\mathrm{B}$.

Quadro 10. Velocidade econômica $\left(\mathrm{m} \mathrm{s}^{-1}\right)$ para a tubulação de aço galvanizado com tempo de funcionamento de 1000,2000 e 3000 horasano $^{-1}$ considerando diferentes tarifas energéticas (Convencional, Verde e Azul), 100\% de aumento nos custos atuais de aquisição da tubulação e desnível geométrico total de $50 \mathrm{~m}$.

\begin{tabular}{|c|c|c|c|c|c|c|c|c|c|}
\hline \multicolumn{10}{|c|}{$100 \%$} \\
\hline \multirow{2}{*}{ Vazão $\left(\mathrm{m}^{3} \mathrm{~h}^{-1}\right)$} & \multicolumn{3}{|c|}{ Convencional } & \multicolumn{3}{|c|}{ Verde } & \multicolumn{3}{|c|}{ Azul } \\
\hline & $1000 \mathrm{~h}$ & $2000 \mathrm{~h}$ & $3000 \mathrm{~h}$ & $1000 \mathrm{~h}$ & $2000 \mathrm{~h}$ & $3000 \mathrm{~h}$ & $1000 \mathrm{~h}$ & $2000 \mathrm{~h}$ & $3000 \mathrm{~h}$ \\
\hline 50 & 1,76 & 1,76 & 1,13 & 1,76 & 1,76 & 1,13 & 1,76 & 1,76 & 1,13 \\
\hline 100 & 2,26 & 2,26 & 2,26 & 2,26 & 2,26 & 2,26 & 2,26 & 2,26 & 2,26 \\
\hline 150 & 2,35 & 2,35 & 1,72 & 2,35 & 2,35 & 1,72 & 2,35 & 2,35 & 1,72 \\
\hline 200 & 2,3 & 1,76 & 1,76 & 2,3 & 1,76 & 1,76 & 2,3 & 1,76 & 1,76 \\
\hline 250 & 2,21 & 2,21 & 2,21 & 2,21 & 2,21 & 2,21 & 2,21 & 2,21 & 2,21 \\
\hline 300 & 1,70 & 1,70 & 1,70 & 2,65 & 2,65 & 2,65 & 2,65 & 2,65 & 2,65 \\
\hline
\end{tabular}

*Valores de velocidade média sombreados correspondem à tarifa de cobrança do grupo A, valores não sombreados grupo B.

$\mathrm{h}$ ano ${ }^{-1}$. Assim como, para a vazão de $100 \mathrm{~m}^{3} \mathrm{~h}^{-1}$ para $3000 \mathrm{~h}$ ano $^{-1}$ e para a vazão de $150 \mathrm{~m}^{3} \mathrm{~h}^{-1}$ nos tempos de funcionamento de 2000 e $3000 \mathrm{~h}$ ano ${ }^{-1}$. Para a vazão de $200 \mathrm{~m}^{3} \mathrm{~h}^{-1} \mathrm{o}$ aumento foi observado em $1000 \mathrm{~h} \mathrm{ano}^{-1}$. Na vazão de $250 \mathrm{~m}^{3}$ $\mathrm{h}^{-1}$ não houve efeito do aumento dado aos custos de aquisição da tubulação sobre a velocidade econômica. Para a vazão de $300 \mathrm{~m}^{3} \mathrm{~h}^{-1}$ foi observado aumento da velocidade econômica, nos tempos de funcionamento de 2000 e $3000 \mathrm{~h} \mathrm{ano}^{-1}$, para o sistema tarifário Verde e Azul. Nota-se também que, para a vazão de $300 \mathrm{~m}^{3} \mathrm{~h}^{-1}$, houve mudança no grupo de cobrança, passando do Grupo B para o Grupo A, nos sistemas tarifários Verde e Azul.

Os acréscimos observados nas velocidades econômicas em alguns valores são justificados pelo aumento no custo de aquisição da tubulação, que contribuem expressivamente com o aumento nos custos fixos, consequentemente um aumento no custo total, assim o diâmetro econômico tende a diminuir, aumentando a velocidade de escoamento.

Nos Quadros 11 e 12 apresentam-se os resultados de velocidade econômica para a tubulação de PVC considerando um acréscimo de 30 e 100\%, respectivamente, nos custos atuais de aquisição da tubulação, permanecendo os custos atuais de energia de bombeamento, tempo de funcionamento de 1000,2000 e $3000 \mathrm{~h} \mathrm{ano}^{-1}$ e diferentes tarifas energéticas.

\section{REVENG


Quadro 11. Velocidade econômica $\left(\mathrm{m} \mathrm{s}^{-1}\right)$ para a tubulação de PVC com tempo de funcionamento de 1000, 2000 e $3000 \mathrm{~h}$ ano $^{-1}$ considerando diferentes tarifas energéticas, $30 \%$ de aumento nos custos atuais de aquisição da tubulação e desnível geométrico total de $50 \mathrm{~m}$.

\begin{tabular}{cccccccccccc}
\hline \multicolumn{10}{c}{$30 \%$} \\
\hline \multirow{2}{*}{ Vazão $\left(\mathrm{m}^{3} \mathrm{~h}^{-1}\right)$} & \multicolumn{3}{c}{ Convencional } & \multicolumn{3}{c}{ Verde } & \multicolumn{3}{c}{ Azul } \\
\cline { 2 - 14 } & $1000 \mathrm{~h}$ & $2000 \mathrm{~h}$ & $3000 \mathrm{~h}$ & & $1000 \mathrm{~h}$ & $2000 \mathrm{~h}$ & $3000 \mathrm{~h}$ & $1000 \mathrm{~h}$ & $2000 \mathrm{~h}$ & $3000 \mathrm{~h}$ \\
\hline 50 & 1,41 & 1,41 & 1,41 & 1,41 & 1,41 & 1,41 & 1,41 & 1,41 & 1,41 \\
100 & 1,36 & 1,36 & 1,36 & 1,36 & 1,36 & 1,36 & 1,36 & 1,36 & 1,36 \\
150 & 2,04 & 1,23 & 1,23 & 2,04 & 1,23 & 1,23 & 2,04 & 1,23 & 1,23 \\
200 & 1,64 & 1,64 & 1,64 & 1,64 & 1,64 & 1,64 & 1,64 & 1,64 & 1,64 \\
250 & 2,05 & 2,05 & 1,34 & 2,05 & 2,05 & 2,05 & 2,05 & 2,05 & 2,05 \\
300 & 1,61 & 1,61 & 1,61 & 1,61 & 1,61 & 1,61 & 1,61 & 1,61 & 1,61 \\
\hline
\end{tabular}

* Valores de velocidade média sombreados correspondem à tarifa de cobrança do grupo A, valores não sombreados grupo $\mathrm{B}$.

Quadro 12. Velocidade econômica $\left(\mathrm{m} \mathrm{s}^{-1}\right)$ para a tubulação de PVC com tempo de funcionamento de 1000, 2000 e $3000 \mathrm{~h} \mathrm{ano}^{-1}$ considerando diferentes tarifas energéticas (Convencional, Verde e Azul), $100 \%$ de aumento nos custos atuais de aquisição da tubulação e desnível geométrico total de $50 \mathrm{~m}$.

\begin{tabular}{ccccccccccc}
\hline \multicolumn{10}{c}{$100 \%$} \\
\hline \multirow{2}{*}{ Vazão $\left(\mathrm{m}^{3} \mathrm{~h}^{-1}\right)$} & \multicolumn{10}{c}{ Convencional } \\
\cline { 2 - 13 } & $1000 \mathrm{~h}$ & $2000 \mathrm{~h}$ & $3000 \mathrm{~h}$ & $1000 \mathrm{~h}$ & $2000 \mathrm{~h}$ & $3000 \mathrm{~h}$ & $1000 \mathrm{~h}$ & $2000 \mathrm{~h}$ & $3000 \mathrm{~h}$ \\
\hline 50 & 1,41 & 1,41 & 1,41 & 1,41 & 1,41 & 1,41 & 1,41 & 1,41 & 1,41 \\
100 & 1,36 & 1,36 & 1,36 & 1,36 & 1,36 & 1,36 & 1,36 & 1,36 & 1,36 \\
150 & 2,04 & 1,23 & 1,23 & 2,04 & 1,23 & 1,23 & 1,23 & 1,23 & 1,23 \\
200 & 1,64 & 1,64 & 1,64 & 1,64 & 1,64 & 1,64 & 1,64 & 1,64 & 1,64 \\
250 & 2,05 & 2,05 & 2,05 & 2,05 & 2,05 & 2,05 & 2,05 & 2,05 & 2,05 \\
300 & 1,61 & 1,61 & 1,61 & 2,46 & 2,46 & 2,46 & 2,46 & 2,46 & 2,46 \\
\hline
\end{tabular}

*Valores de velocidade média sombreados correspondem à tarifa de cobrança do grupo A, valores não sombreados grupo B.

Observando os resultados das Tabelas $11 \mathrm{e}$ 12, para tubulação de PVC, para as vazões de $50,100,150,200$ e $250 \mathrm{~m}^{3} \mathrm{~h}^{-1}$, nota-se que não houve variação da velocidade econômica em razão dos aumentos dados nos custos de aquisição da tubulação. Foi observado apenas aumento da velocidade econômica para a vazão de $250 \mathrm{~m}^{3} \mathrm{~h}^{-1}$ nos sistemas tarifários Verde e Azul, para todos os tempos de funcionamento.

Comparando os resultados com os obtidos para a tubulação de PVC com os obtidos para a tubulação de aço galvanizado, nota-se que a tubulação de PVC foi menos sensível ao aumento nos custos de aquisição da tubulação. Fato que pode ser explicado pelo custo unitário de aquisição da tubulação de PVC, de mesmo diâmetro, ser menor.
Assim, o reflexo nos custos finais para a tubulação de PVC será menor, sendo necessário um aporte maior na variação dos custos de aquisição para refletir na velocidade econômica.

Segundo Perroni et al. (2015), variações de velocidade econômica em adutoras de PVC não apresentam diferenças acentuadas entre si, quando considerados comprimentos variando de 100 a $2000 \mathrm{~m}$, independente da variação da relação de custos energia/tubulação. No entanto, em adutoras de aço galvanizado a variação é maior, pois são maiores os custos de investimento na aquisição dos tubos de aço, bem como, a perda de carga, conferem uma maior sensibilidade às variações de custos da energia e da tubulação.

Os valores de velocidade econômica encontrados 
nas diversas situações analisadas variaram entre 0,88 e 2,65 m.s. s $^{-1}$ para tubulações de aço galvanizado e entre 0,68 e 2,54 m. $\mathrm{s}^{-1}$ para tubulações de PVC, valores estes próximos dos apresentados em literatura, que consideram velocidades de até 2,6 m.s. ${ }^{-1}$, como apresentado por Azevedo Netto et al. (2002) e Bernardo et al. (2006).

Observa-se que o valor da velocidade econômica é decrescente com aumento no tempo de funcionamento, seja para tubulações de aço galvanizado ou para tubulações de PVC. Este comportamento pode ser explicado devido ao aumento expressivo no custo com energia de bombeamento (custo variável) que está diretamente relacionado à perda de carga, sendo que, para diminuição da perda de carga e dos custos provenientes da mesma, é necessário um aumento no diâmetro da tubulação, proporcionando velocidades econômicas menores.

Verificando-se a influência do comprimento da adutora na velocidade econômica, confirmaramse os resultados obtidos por Perroni (2008), que analisou a influência do comprimento da adutora na velocidade econômica para um mesmo tipo de material da tubulação e tempo de funcionamento, e concluiu que não há influência do comprimento da adutora no valor final da velocidade econômica, pois a variação do comprimento da adutora reflete diretamente nos custos fixos e também nos custos variáveis, não alterando o diâmetro econômico independente dos valores de comprimento.

Comparando-se valores de velocidade econômica entre tubulações de aço galvanizado e tubulações de PVC, observa-se que os valores para tubulações de aço galvanizado são ligeiramente superiores aos obtidos para tubulação de PVC. Este fato pode ser explicado devido ao custo unitário de aquisição do tubo de aço galvanizado, de mesmo diâmetro, ser superior ao custo do tubo de PVC, sendo que, para uma mesma vazão, é mais econômico utilizar um tubo de aço galvanizado de menor diâmetro ou um tubo de maior diâmetro para tubulação de PVC.

\section{CONCLUSÃO}

- Para tubulação de aço galvanizado a velocidade econômica variou entre 0,88 e $2,65 \mathrm{~m} \mathrm{~s}^{-1}$ e para tubulação de PVC entre 0,68 e $2,54 \mathrm{~m} \mathrm{~s}^{-1}$. O valor da velocidade econômica foi decrescente com o aumento no tempo de funcionamento da adutora e o comprimento da adutora não influência na velocidade econômica.

- Aumento no desnível geométrico total provocou diminuição na velocidade econômica e mudança no grupo de cobrança de tarifa energética; com o aumento da tarifa energética, ocorreu uma diminuição da velocidade econômica e,com o aumento do custo da tubulação, ocorreu um aumento da velocidade econômica.

\section{REFFERÊNCIAS BIBLIOGRÁFICAS}

AGÊNCIA NACIONAL DAS ÁGUAS. Conjuntura dos recursos hídricos no Brasil: Informe 2014. 105p. Brasília, 2015. Disponível em: $<$ http://conjuntura.ana.gov.br/docs/conj2014 inf.pdf> Acesso: 12 fev. 2016.

AGÊNCIA NACIONAL DE ENERGIA ELÉTRICA. Tarifas de fornecimento de energia elétrica. Brasília: 2005. (Cadernos temáticos). Disponível em: $\quad<$ http://www.aneel.gov.br/ arquivos/pdf/caderno4capa.pdf $>$. Acesso: 08 nov. 2014.

AGÊNCIA NACIONAL DE ENERGIA ELÉTRICA. Bandeiras tarifárias. Disponível em: $<$ http://www.aneel.gov.br/area.cfm?idArea=758 $>$. Acesso: 08 nov. 2014.

AZEVEDO NETTO, J.M.; FERNANDES, M.F.; ARAUJO, R.; ITO, A.E. Manual de hidráulica. 8 ed. São Paulo: E. Blücher, 2002. 669p.

AZEVEDO, R.C.M. de; SRINIVASAN, V.S.; GAVAZZA, S.; SANTOS, S.M. dos. O papel dos recursos hídricos no desenvolvimento sustentável do nordeste. Simpósio Brasileiro de Recursos Hídricos. Bento Gonçalves, Rio Grande do Sul. 17 a 23 de Novembro de 2013. 8p.

BERNARDO, S.; SOARES, A.A.; MANTOVANI, E.C. Manual de irrigação. 8 ed. Viçosa, MG: UFV, 2006. 625p.

\section{REVENG}


CAIXA ECONÔMICA FEDERAL. Índices da construção civil. Disponível em: $<$ http://wwwl. caixa.gov.br/gov/gov_social/municipal/programa des urbano/SINAPI/index.asp>. Acesso: 14 nov. 2014.

CARVALHO, J.A.; REIS, J.B.R S. Avaliação dos custos de energia de bombeamento e determinação do diâmetro econômico da tubulação. Ciência e Agrotecnologia, Lavras, v.24, n.2, p.411-449, abr./ jun. 2000.

COMPANHIA ENERGÉTICA DE MINAS GERAIS. Valores de tarifas e serviços. Disponível em: $<$ http://www.cemig.com.br/pt-br/atendimento/ Paginas/valores de tarifa e servicos.aspx $>$. Acesso: 20 nov. 2014.

DERCAS, N.; VALIANTZAS, J. D. Two explicit optimum design methods for a simple irrigation delivery system: comparative application. Irrigation and Drainage. v.61, p.10-19, abr. 2012.

DONG, H.; GENG, Y.; SARKIS, J.; OKADERA, T.; XUE, B. Regional Water Footprint Evaluation in China: A case of Liaoning. H. Science of the Total Environment, v.442, p.215-224, jan. 2013.

FREIRE, P.K.C. Estudo comparativo entre metodologias de dimensionamento econômico de adutoras. 2000. 120f. Dissertação (Mestrado em Engenharia Civil) - Universidade Federal da Paraíba. Campina Grande, 2000.

GOMES, H.P.; SILVA, J.G. Dimensionamento econômico de sistemas de distribuição de água, considerando variáveis as condições de contorno do projeto. Revista Brasileira de Recursos Hídricos. Porto Alegre, v.11, n.2, abr/jun 2006, p.99-110.

LIMA, A.C.; GUIMARÃES JÚNIOR, S.C.; FIETZ, C.R; CAMACHO, J.R. Avaliação e análise da eficiência energética na irrigação em sistemas de pivô central. Revista Brasileira de Engenharia Agrícola e Ambiental, v.13, n.4, jul./ago., 2009.
MARCUZZO,
F.F.N.;
WENDLAND,

E. Otimização de rede de irrigação de microaspersão usando algoritmos genéticos sob diferentes declividades e tarifação de água e energia elétrica. Engenharia na Agricultura, Viçosa, MG, v.18, n.1, jan/fev. 2010, p.50-62.

MELO, J.F. Custos da irrigação por aspersão em Minas Gerais. 1993. 147f. Dissertação (Mestrado em Engenharia Agrícola) - Universidade Federal de Viçosa, Viçosa, MG, 1993.

PERRONI, B.L.T. Velocidade econômica de escoamento e composição de custos de sistemas de irrigação por aspersão. 2008. 93f. Dissertação (Mestrado em Engenharia Agrícola) - Universidade Federal de Lavras, Lavras, MG, 2008.

PERRONI, B.L.T.; CARVALHO, J.A.; FARIA, L.C. Velocidade de escoamento e custos de energia de bombeamento. Engenharia Agrícola, Jaboticabal, v.31, n.3, mai/jun, 2011, p.487-496.

PERRONI, B.L.T.; FARIA, L.C.; CARVALHO, J.A.; OLIVEIRA, H.F.E. Influência do custo da energia elétrica e do material da tubulação na velocidade econômica de bombeamentos. Irriga, Botucatu, v.20, n.1, jan/mar, 2015, p.13-20.

PIRES, M.A.A. Análise da evolução da eficiência das empresas distribuidoras de energia elétrica no Brasil, após a reestruturação do setor, no período de 2001 a 2005. 2008. 179f. Dissertação (Mestrado em Economia) - Universidade Federal de Pernambuco, Recife, 2008.

PROGRAMA NACIONAL DE IRRIGAÇÃO. Tempo de irrigar: manual do irrigante. São Paulo: Mater, Fundação Victor Civita. 1987. 160p.

SANTOS, G. J.dos;MARION, J.C. Administração de custos na agropecuária. $2^{\circ}$ ed. São Paulo: Atlas, 1996. 139p.

SOUZA, I.H.; ANDRADE, E.M.; SILVA, E.L. Avaliação hidráulica de um sistema de irrigação localizada de baixa pressão, projetado pelo software "bubbler". Engenharia Agrícola, Jaboticabal, v.25, n.1, 2005. p.264-271. 
ZOCOLER, J.L.; Filho, F.C.B.; Oliveira, L.A.F.; Hernadez, F.B.T. Modelo para determinação do diâmetro e velocidade de escoamento econômica em sistemas elevatórios de água. $3^{\circ}$ Congresso Temático de Dinâmica e Controle da SBMAC. 31 mai. a 3 jun. de 2004. Anais.., 2004 UNESP. Ilha Solteira, SP.
ZOCOLER, J.L.; HERNANDEZ, F.B.T.; SAAD, J.C.C.; CRUZ, R.L. Período de recuperação do investimento em bomba hidráulica de melhor rendimento em sistemas de bombeamento na tarifa horossazonal verde. Engenharia Agrícola, Jaboticabal, v.31, n.2, 2011, p.290-302. 